\title{
Strong and Weak Convergence for Asymptotically Almost Negatively Associated Random Variables
}

\author{
Aiting Shen and Ranchao Wu \\ School of Mathematical Science, Anhui University, Hefei 230039, China \\ Correspondence should be addressed to Aiting Shen; empress201010@126.com
}

Received 10 December 2012; Accepted 16 January 2013

Academic Editor: Binggen Zhang

Copyright ( 2013 A. Shen and R. Wu. This is an open access article distributed under the Creative Commons Attribution License, which permits unrestricted use, distribution, and reproduction in any medium, provided the original work is properly cited.

\begin{abstract}
The strong law of large numbers for sequences of asymptotically almost negatively associated (AANA, in short) random variables is obtained, which generalizes and improves the corresponding one of Bai and Cheng (2000) for independent and identically distributed random variables to the case of AANA random variables. In addition, the Feller-type weak law of large number for sequences of AANA random variables is obtained, which generalizes the corresponding one of Feller (1946) for independent and identically distributed random variables.
\end{abstract}

\section{Introduction}

Many useful linear statistics based on a random sample are weighted sums of independent and identically distributed random variables. Examples include least-squares estimators, nonparametric regression function estimators, and jackknife estimates,. In this respect, studies of strong laws for these weighted sums have demonstrated significant progress in probability theory with applications in mathematical statistics.

Let $\left\{X_{n}, n \geq 1\right\}$ be a sequence of random variables and let $\left\{a_{n i}, 1 \leq i \leq n, n \geq 1\right\}$ be an array of constants. A common expression for these linear statistics is $T_{n}=\sum_{i=1}^{n} a_{n i} X_{i}$. Some recent results on the strong law for linear statistics $T_{n}$ can be found in Cuzick [1], Bai et al. [2], Bai and Cheng [3], Cai [4], Wu [5], Sung [6], Zhou et al. [7], and Wang et al. [8]. Our emphasis in this paper is focused on the result of Bai and Cheng [3]. They gave the following theorem.

Theorem A. Suppose that $1<\alpha, \beta<\infty, 1 \leq p<2$, and $1 / p=1 / \alpha+1 / \beta$. Let $\left\{X, X_{n}, n \geq 1\right\}$ be a sequence of independent and identically distributed random variables satisfying $E X=0$, and let $\left\{a_{n k}, 1 \leq k \leq n, n \geq 1\right\}$ be an array of real constants such that

$$
\limsup _{n \rightarrow \infty}\left(\frac{1}{n} \sum_{k=1}^{n}\left|a_{n k}\right|^{\alpha}\right)^{1 / \alpha}<\infty .
$$

If $E|X|^{\beta}<\infty$, then

$$
\lim _{n \rightarrow \infty} n^{-1 / p} \sum_{k=1}^{n} a_{n k} X_{k}=0 \quad \text { a.s. }
$$

We point out that the independence assumption is not plausible in many statistical applications. So it is of interest to extend the concept of independence to the case of dependence. One of these dependence structures is asymptotically almost negatively associated, which was introduced by Chandra and Ghosal [9] as follows.

Definition 1. A sequence $\left\{X_{n}, n \geq 1\right\}$ of random variables is called asymptotically almost negatively associated (AANA, in short) if there exists a nonnegative sequence $u(n) \rightarrow 0$ as $n \rightarrow \infty$ such that

$$
\begin{aligned}
& \operatorname{Cov}\left(f\left(X_{n}\right), g\left(X_{n+1}, X_{n+2}, \ldots, X_{n+k}\right)\right) \\
& \quad \leq u(n)\left[\operatorname{Var}\left(f\left(X_{n}\right)\right) \operatorname{Var}\left(g\left(X_{n+1}, X_{n+2}, \ldots, X_{n+k}\right)\right)\right]^{1 / 2},
\end{aligned}
$$

for all $n, k \geq 1$ and for all coordinatewise nondecreasing continuous functions $f$ and $g$ whenever the variances exist.

It is easily seen that the family of AANA sequence contains negatively associated (NA, in short) sequences (with $u(n)=0, n \geq 1)$ and some more sequences of random 
variables which are not much deviated from being negatively associated. An example of an AANA sequence which is not NA was constructed by Chandra and Ghosal [9]. Hence, extending the limit properties of independent or NA random variables to the case of AANA random variables is highly desirable in the theory and application.

Since the concept of AANA sequence was introduced by Chandra and Ghosal [9], many applications have been found. See, for example, Chandra and Ghosal [9] derived the Kolmogorov type inequality and the strong law of large numbers of Marcinkiewicz-Zygmund; Chandra and Ghosal [10] obtained the almost sure convergence of weighted averages; Wang et al. [11] established the law of the iterated logarithm for product sums; Ko et al. [12] studied the HájekRényi type inequality; Yuan and An [13] established some Rosenthal type inequalities for maximum partial sums of AANA sequence; Wang et al. [14] obtained some strong growth rate and the integrability of supremum for the partial sums of AANA random variables; Wang et al. $[15,16]$ studied complete convergence for arrays of rowwise AANA random variables and weighted sums of arrays of rowwise AANA random variables, respectively; $\mathrm{Hu}$ et al. [17] studied the strong convergence properties for AANA sequence; Yang et al. [18] investigated the complete convergence, complete moment convergence, and the existence of the moment of supermum of normed partial sums for the moving average process for AANA sequence, and so forth.

The main purpose of this paper is to study the strong convergence for AANA random variables, which generalizes and improves the result of Theorem A. In addition, we will give the Feller-type weak law of large number for sequences of AANA random variables, which generalizes the corresponding one of Feller [19] for independent and identically distributed random variables.

Throughout this paper, let $\left\{X_{n}, n \geq 1\right\}$ be a sequence of AANA random variables with the mixing coefficients $\{u(n), n \geq 1\} . S_{n}=\sum_{i=1}^{n} X_{i}$. For $s>1$, let $t \doteq s /(s-1)$ be the dual number of $s$. The symbol $C$ denotes a positive constant which may be different in various places. Let $I(A)$ be the indicator function of the set $A . a_{n}=O\left(b_{n}\right)$ stands for $a_{n} \leq C b_{n}$.

The definition of stochastic domination will be used in the paper as follows.

Definition 2. A sequence $\left\{X_{n}, n \geq 1\right\}$ of random variables is said to be stochastically dominated by a random variable $X$ if there exists a positive constant $C$ such that

$$
P\left(\left|X_{n}\right|>x\right) \leq C P(|X|>x),
$$

for all $x \geq 0$ and $n \geq 1$.

Our main results are as follows.

Theorem 3. Suppose that $0<\alpha, \beta<\infty, 0<p<2$, and $1 / p=1 / \alpha+1 / \beta$. Let $\left\{X_{n}, n \geq 1\right\}$ be a sequence of AANA random variables, which is stochastically dominated by a random variable $X$ and $E X_{n}=0$, if $\beta>1$. Suppose that there exists a positive integer $k$ such that $\sum_{n=1}^{\infty} u^{1 /(1-s)}(n)<\infty$ for some $s \in\left(3 \cdot 2^{k-1}, 4 \cdot 2^{k-1}\right]$ and $s>1 /(\min \{1 / 2,1 / \alpha, 1 / \beta, 1 / p-$ $1 / 2\})$. Let $\left\{a_{n i}, i \geq 1, n \geq 1\right\}$ be an array of real constants satisfying

$$
\sum_{i=1}^{n}\left|a_{n i}\right|^{\alpha}=O(n)
$$

If $E|X|^{\beta}<\infty$, then

$$
\lim _{n \rightarrow \infty} n^{-1 / p} \max _{1 \leq j \leq n}\left|\sum_{i=1}^{j} a_{n i} X_{i}\right|=0 \quad \text { a.s. }
$$

Remark 4. Theorem 3 generalizes and improves Theorem A of Bai and Cheng [3] for independent and identically distributed random variables to the case of AANA random variables, since Theorem 3 removes the identically distributed condition and expands the ranges $\alpha, \beta$, and $p$, respectively.

At last, we will present the Feller-type weak law of large number for sequences of AANA random variables, which generalizes the corresponding one of Feller [19] for independent and identically distributed random variables.

Theorem 5. Let $\alpha>1 / 2$ and $\left\{X, X_{n}, n \geq 1\right\}$ be a sequence of identically distributed AANA random variables with the mixing coefficients $\{u(n), n \geq 1\}$ satisfying $\sum_{n=1}^{\infty} u^{2}(n)<\infty$. If

$$
\lim _{n \rightarrow \infty} n P\left(|X|>n^{\alpha}\right)=0,
$$

then

$$
\frac{S_{n}}{n^{\alpha}}-n^{1-\alpha} E X I\left(|X| \leq n^{\alpha}\right) \stackrel{P}{\rightarrow} 0 .
$$

\section{Preparations}

To prove the main results of the paper, we need the following lemmas. The first two lemmas were provided by Yuan and An [13].

Lemma 6 (cf. see [13, Lemma 2.1]). Let $\left\{X_{n}, n \geq 1\right\}$ be a sequence of $A A N A$ random variables with mixing coefficients $\{u(n), n \geq 1\}, f_{1}, f_{2}, \ldots$ be all nondecreasing (or all nonincreasing) continuous functions, then $\left\{f_{n}\left(X_{n}\right), n \geq 1\right\}$ is still a sequence of AANA random variables with mixing coefficients $\{u(n), n \geq 1\}$.

Lemma 7 (cf. see [13, Theorem 2.1]). Let $p>1$ and $\left\{X_{n}, n \geq\right.$ $1\}$ be a sequence of zero mean random variables with mixing coefficients $\{u(n), n \geq 1\}$.

If $\sum_{n=1}^{\infty} u^{2}(n)<\infty$, then there exists a positive constant $C_{p}$ depending only on $p$ such that for all $n \geq 1$ and $1<p \leq 2$,

$$
E\left(\max _{1 \leq j \leq n}\left|\sum_{i=1}^{j} X_{i}\right|^{p}\right) \leq C_{p} \sum_{i=1}^{n} E\left|X_{i}\right|^{p} .
$$


If $\sum_{n=1}^{\infty} u^{1 /(p-1)}(n)<\infty$ for some $p \in\left(3 \cdot 2^{k-1}, 4 \cdot 2^{k-1}\right]$, where integer number $k \geq 1$, then there exists a positive constant $D_{p}$ depending only on $p$ such that for all $n \geq 1$,

$$
E\left(\max _{1 \leq j \leq n}\left|\sum_{i=1}^{j} X_{i}\right|^{p}\right) \leq D_{p}\left\{\sum_{i=1}^{n} E\left|X_{i}\right|^{p}+\left(\sum_{i=1}^{n} E X_{i}^{2}\right)^{p / 2}\right\} .
$$

The last one is a fundamental property for stochastic domination. The proof is standard, so the details are omitted.

Lemma 8. Let $\left\{X_{n}, n \geq 1\right\}$ be a sequence of random variables, which is stochastically dominated by a random variable $X$. Then for any $\alpha>0$ and $b>0$,

$$
\begin{gathered}
E\left|X_{n}\right|^{\alpha} I\left(\left|X_{n}\right| \leq b\right) \\
\leq C_{1}\left[E|X|^{\alpha} I(|X| \leq b)+b^{\alpha} P(|X|>b)\right], \\
E\left|X_{n}\right|^{\alpha} I\left(\left|X_{n}\right|>b\right) \leq C_{2} E|X|^{\alpha} I(|X|>b),
\end{gathered}
$$

where $C_{1}$ and $C_{2}$ are positive constants.

\section{Proofs of the Main Results}

Proof of Theorem 3. Without loss of generality, we assume that $a_{n i} \geq 0$ (otherwise, we use $a_{n i}^{+}$and $a_{n i}^{-}$instead of $a_{n i}$, and note that $\left.a_{n i}=a_{n i}^{+}-a_{n i}^{-}\right)$. Denote for $1 \leq i \leq n$ and $n \geq 1$ that

$$
\begin{gathered}
Y_{i}=-n^{1 / \beta} I\left(X_{i}<-n^{1 / \beta}\right) \\
+X_{i} I\left(\left|X_{i}\right| \leq n^{1 / \beta}\right)+n^{1 / \beta} I\left(X_{i}>n^{1 / \beta}\right), \\
Z_{i}=\left(X_{i}+n^{1 / \beta}\right) I\left(X_{i}<-n^{1 / \beta}\right)+\left(X_{i}-n^{1 / \beta}\right) I\left(X_{i}>n^{1 / \beta}\right) .
\end{gathered}
$$

Hence, $X_{i}=Y_{i}+Z_{i}$, which implies that

$$
\begin{aligned}
n^{-1 / p} \max _{1 \leq j \leq n}\left|\sum_{i=1}^{j} a_{n i} X_{i}\right| \leq & n^{-1 / p} \max _{1 \leq j \leq n}\left|\sum_{i=1}^{j} a_{n i} Z_{i}\right| \\
& +n^{-1 / p} \max _{1 \leq j \leq n}\left|\sum_{i=1}^{j} a_{n i} Y_{i}\right| \\
\leq & n^{-1 / p} \max _{1 \leq j \leq n}\left|\sum_{i=1}^{j} a_{n i} Z_{i}\right| \\
& +n^{-1 / p} \max _{1 \leq j \leq n}\left|\sum_{i=1}^{j} a_{n i} E Y_{i}\right| \\
& +n^{-1 / p} \max _{1 \leq j \leq n} \mid \sum_{i=1}^{j} a_{n i}\left(Y_{i}-E Y_{i}\right) \\
\doteq & H+I+J .
\end{aligned}
$$

To prove (6), it suffices to show that $H \rightarrow 0$ a.s., $I \rightarrow 0$ and $J \rightarrow 0$ a.s. as $n \rightarrow \infty$.

Firstly, we will show that $H \rightarrow 0$ a.s.

For any $0<\gamma \leq \alpha$, it follows from (5) and Hölder's inequality that

$$
\sum_{i=1}^{n}\left|a_{n i}\right|^{\gamma} \leq\left(\sum_{i=1}^{n}\left|a_{n i}\right|^{\alpha}\right)^{\gamma / \alpha} \times\left(\sum_{i=1}^{n} 1\right)^{1-\gamma / \alpha} \leq C n
$$

for any $0<\alpha \leq \gamma$, it follows from (5) again that

$$
\sum_{i=1}^{n}\left|a_{n i}\right|^{\gamma} \leq\left(\sum_{i=1}^{n}\left|a_{n i}\right|^{\alpha}\right)^{\gamma / \alpha} \leq C n^{\gamma / \alpha}
$$

Combining (14) and (15), we have

$$
\sum_{i=1}^{n}\left|a_{n i}\right|^{\gamma} \leq C n^{\max (1, \gamma / \alpha)}
$$

The condition $E|X|^{\beta}<\infty$ yields that

$$
\begin{aligned}
\sum_{n=1}^{\infty} P\left(Z_{n} \neq 0\right) & =\sum_{n=1}^{\infty} P\left(\left|X_{n}\right|>n^{1 / \beta}\right) \\
& \leq C \sum_{n=1}^{\infty} P\left(|X|>n^{1 / \beta}\right) \leq C E|X|^{\beta}<\infty,
\end{aligned}
$$

which implies that $P\left(Z_{n} \neq 0\right.$, i.o. $)=0$ by Borel-Cantelli lemma. Thus, we have by (5) that

$$
\begin{aligned}
H & \doteq n^{-1 / p} \max _{1 \leq j \leq n}\left|\sum_{i=1}^{j} a_{n i} Z_{i}\right| \\
& \leq n^{-1 / p} \sum_{i=1}^{n}\left|a_{n i} Z_{i}\right| \\
& \leq C n^{-1 / p}\left(\max _{1 \leq i \leq n}\left|a_{n i}\right|^{\alpha}\right)^{1 / \alpha} \sum_{i=1}^{n}\left|Z_{i}\right| \\
& \leq C n^{-1 / p}\left(\sum_{i=1}^{n}\left|a_{n i}\right|^{\alpha}\right)^{1 / \alpha} \sum_{i=1}^{n}\left|Z_{i}\right| \\
& \leq C n^{-1 / \beta} \sum_{i=1}^{n}\left|Z_{i}\right| \longrightarrow 0 \text { a.s., as } n \longrightarrow \infty .
\end{aligned}
$$

Secondly, we will prove that

$$
I \doteq n^{-1 / p} \max _{1 \leq j \leq n}\left|\sum_{i=1}^{j} a_{n i} E Y_{i}\right| \longrightarrow 0, \quad \text { as } n \longrightarrow \infty .
$$


If $0<\beta \leq 1$, then we have by Lemma 8 and (16) that

$$
\begin{aligned}
& I \leq n^{-1 / p} \sum_{i=1}^{n}\left|a_{n i} E Y_{i}\right| \\
& \leq n^{-1 / p} \sum_{i=1}^{n}\left|a_{n i}\right|\left[E\left|X_{i}\right| I\left(\left|X_{i}\right| \leq n^{1 / \beta}\right)\right. \\
& \left.\quad+n^{1 / \beta} P\left(\left|X_{i}\right|>n^{1 / \beta}\right)\right] \\
& \leq C n^{-1 / p} \sum_{i=1}^{n}\left|a_{n i}\right|\left[E|X| I\left(|X| \leq n^{1 / \beta}\right)\right. \\
& \left.\quad+n^{1 / \beta} P\left(|X|>n^{1 / \beta}\right)\right] \\
& \leq C n^{-1 / p} \sum_{i=1}^{n}\left|a_{n i}\right|\left[n^{(1-\beta) / \beta} E|X|^{\beta} I\left(|X| \leq n^{1 / \beta}\right)\right. \\
& \left.\quad+n^{1 / \beta-1} E|X|^{\beta} I\left(|X|>n^{1 / \beta}\right)\right] \\
& \leq C n^{-1 / \alpha-1} E|X|^{\beta} \sum_{i=1}^{n}\left|a_{n i}\right| \quad \text { as } n \longrightarrow \infty .
\end{aligned}
$$

If $\beta>1$, then we have by $E X_{n}=0$, Lemma 8 and (16) that

$$
\begin{aligned}
I \leq & n^{-1 / p} \sum_{i=1}^{n}\left|a_{n i} E Y_{i}\right| \\
\leq & C n^{-1 / p} \sum_{i=1}^{n}\left|a_{n i}\right| \\
& \times\left[E\left|X_{i}\right| I\left(\left|X_{i}\right|>n^{1 / \beta}\right)+n^{1 / \beta} P\left(\left|X_{i}\right|>n^{1 / \beta}\right)\right] \\
\leq & C n^{-1 / p} \sum_{i=1}^{n}\left|a_{n i}\right| E|X| I\left(|X|>n^{1 / \beta}\right) \\
\leq & C n^{-1 / p} \sum_{i=1}^{n}\left|a_{n i}\right| n^{1 / \beta-1} E|X|^{\beta} I\left(|X|>n^{1 / \beta}\right) \\
\leq & C n^{-1 / \alpha-1+\max (1,1 / \alpha)} \longrightarrow 0, \quad \text { as } n \longrightarrow \infty .
\end{aligned}
$$

Hence, (19) follows from (20) and (21) immediately.

To prove (6), it suffices to show that

$$
H \doteq n^{-1 / p} \max _{1 \leq j \leq n}\left|\sum_{i=1}^{j} a_{n i}\left(Y_{i}-E Y_{i}\right)\right| \longrightarrow 0 \text { a.s., } \quad \text { as } n \longrightarrow \infty \text {. }
$$

By Borel-Cantelli Lemma, we only need to show that for any $\varepsilon>0$,

$$
\sum_{n=1}^{\infty} P\left(\max _{1 \leq j \leq n}\left|\sum_{i=1}^{j} a_{n i}\left(Y_{i}-E Y_{i}\right)\right|>\varepsilon n^{1 / p}\right)<\infty
$$

For fixed $n \geq 1$, it is easily seen that $\left\{a_{n i}\left(Y_{i}-E Y_{i}\right), 1 \leq\right.$ $i \leq n\}$ are still AANA random variables by Lemma 6 . Taking $s>1 / \min \{1 / 2,1 / \alpha, 1 / \beta, 1 / p-1 / 2\}>2$, we have by Markov's inequality and Lemma 7 that

$$
\begin{aligned}
\sum_{n=1}^{\infty} P\left(\max _{1 \leq j \leq n}\left|\sum_{i=1}^{j} a_{n i}\left(Y_{i}-E Y_{i}\right)\right|>\varepsilon n^{1 / p}\right) \\
\leq C \sum_{n=1}^{\infty} n^{-s / p} E\left(\max _{1 \leq j \leq n}\left|\sum_{i=1}^{j} a_{n i}\left(Y_{i}-E Y_{i}\right)\right|^{s}\right) \\
\leq C \sum_{n=1}^{\infty} n^{-s / p} \sum_{i=1}^{n} E\left|a_{n i}\left(Y_{i}-E Y_{i}\right)\right|^{s} \\
\quad+C \sum_{n=1}^{\infty} n^{-s / p}\left(\sum_{i=1}^{n} E\left|a_{n i}\left(Y_{i}-E Y_{i}\right)\right|^{2}\right)^{s / 2} \\
\doteq J_{1}+J_{2} .
\end{aligned}
$$

For $J_{1}$, we have by $C_{r}$ inequality, Jensen's inequality, (15), and Lemma 8 that

$$
\begin{aligned}
J_{1} \leq & C \sum_{n=1}^{\infty} n^{-s / p} \sum_{i=1}^{n}\left|a_{n i}\right|^{s} E\left|Y_{i}\right|^{s} \\
\leq & C \sum_{n=1}^{\infty} n^{-s / p} \sum_{i=1}^{n}\left|a_{n i}\right|^{s} \\
& \times\left[E\left|X_{i}\right|^{s} I\left(\left|X_{i}\right|^{\leq} n^{1 / \beta}\right)+n^{s / \beta} P\left(\left|X_{i}\right|>n^{1 / \beta}\right)\right] \\
\leq & C \sum_{n=1}^{\infty} n^{-s / p} \sum_{i=1}^{n}\left|a_{n i}\right|^{s} \\
& \times\left[E|X|^{s} I\left(|X| \leq n^{1 / \beta}\right)+n^{s / \beta} P\left(|X|>n^{1 / \beta}\right)\right] \\
\leq & C \sum_{n=1}^{\infty} n^{-s / \beta} E|X|^{s} I\left(|X| \leq n^{1 / \beta}\right)+C \sum_{n=1}^{\infty} P\left(|X|>n^{1 / \beta}\right) \\
\leq & C \sum_{n=1}^{\infty} n^{-s / \beta} \sum_{i=1}^{n} E|X|^{s} I \\
\leq & C E|X|^{\beta}<\infty . \\
& \times\left((i-1)^{1 / \beta}<|X| \leq i^{1 / \beta}\right)+C E|X|^{\beta} \\
\leq & C \sum_{i=1}^{\infty} E|X|^{s} I\left((i-1)^{1 / \beta}<|X| \leq i^{1 / \beta}\right) \\
& \times \sum_{n=i}^{\infty} n^{-s / \beta}+C E|X|^{\beta} \\
& \left.\times(i-1)^{1 / \beta}<|X| \leq i^{1 / \beta}\right) i^{-s / \beta+1}+C E|X|^{\beta} \\
& \left(\left.i X\right|^{\beta} I\right. \\
& \\
&
\end{aligned}
$$


Next, we will prove that $J_{2}<\infty$. By $C_{r}$ inequality, Jensen's inequality and Lemma 8 again, we can see that

$$
\begin{array}{rl}
\sum_{i=1}^{n} & E\left|a_{n i}\left(Y_{i}-E Y_{i}\right)\right|^{2} \\
\leq & \sum_{i=1}^{n} a_{n i}^{2} E Y_{i}^{2} \\
\leq & C \sum_{i=1}^{n} a_{n i}^{2}\left[E X_{i}^{2} I\left(\left|X_{i}\right| \leq n^{1 / \beta}\right)+n^{2 / \beta} P\left(\left|X_{i}\right|>n^{1 / \beta}\right)\right] \\
\leq & C \sum_{i=1}^{n} a_{n i}^{2}\left[E X^{2} I\left(|X| \leq n^{1 / \beta}\right)+n^{2 / \beta} P\left(|X|>n^{1 / \beta}\right)\right] \\
\leq & C n^{\max (1,2 / \alpha)} \\
\quad & \times\left[E X^{2} I\left(|X| \leq n^{1 / \beta}\right)+n^{2 / \beta} P\left(|X|>n^{1 / \beta}\right)\right] .
\end{array}
$$

It follows by Markov's inequality and the fact $E|X|^{\beta}<\infty$ that

$$
\begin{aligned}
E X^{2} I & \left(|X| \leq n^{1 / \beta}\right)+n^{2 / \beta} P\left(|X|>n^{1 / \beta}\right) \\
& \leq\left\{\begin{array}{cc}
n^{(2-\beta) / \beta} E|X|^{\beta} I\left(|X| \leq n^{1 / \beta}\right) \\
+n^{-1+2 / \beta} E|X|^{\beta}\left(|X|>n^{1 / \beta}\right), & \beta<2 \\
E X^{2} I\left(|X| \leq n^{1 / \beta}\right)+E X^{2}, & \beta \geq 2,
\end{array}\right. \\
& \leq \begin{cases}C n^{-1+2 / \beta} E|X|^{\beta}, & \beta<2, \\
C E X^{2}, & \beta \geq 2 .\end{cases}
\end{aligned}
$$

If we denote $\delta=\max \{-1+2 / p, 2 / \beta, 2 / \alpha, 1\}$, then we can get by (26) and (27) that

$$
\sum_{i=1}^{n} E\left|a_{n i}\left(Y_{i}-E Y_{i}\right)\right|^{2} \leq C n^{\delta}
$$

It is easily seen that

$$
\begin{aligned}
\left(-\frac{1}{p}+\frac{\delta}{2}\right) s & =\max \left\{-\frac{1}{2},-\frac{1}{\alpha},-\frac{1}{\beta},-\frac{1}{p}+\frac{1}{2}\right\} s \\
& =-\min \left\{\frac{1}{2}, \frac{1}{\alpha}, \frac{1}{\beta}, \frac{1}{p}-\frac{1}{2}\right\} s<-1
\end{aligned}
$$

Hence, we have by (28) and (29) that

$$
J_{2} \leq C \sum_{n=1}^{\infty} n^{(-1 / p+\delta / 2) s}<\infty
$$

which together with $J_{1}<\infty$ yields (23). This completes the proof of the theorem.
Proof of Theorem 5. Denote for $1 \leq i \leq n$ and $n \geq 1$ that

$$
\begin{aligned}
Y_{n i}= & -n^{\alpha} I\left(X_{i}<-n^{\alpha}\right)+X_{i} I \\
& \times\left(\left|X_{i}\right| \leq n^{\alpha}\right)+n^{\alpha} I\left(X_{i}>n^{\alpha}\right)
\end{aligned}
$$

and $T_{n}=\sum_{i=1}^{n} Y_{n i}$. By the assumption (7), we have for any $\varepsilon>0$ that

$$
\begin{aligned}
P\left(\left|\frac{S_{n}}{n^{\alpha}}-\frac{T_{n}}{n^{\alpha}}\right|>\varepsilon\right) & \leq P\left(S_{n} \neq T_{n}\right) \\
& \leq P\left(\bigcup_{i=1}^{n}\left(X_{i} \neq Y_{n i}\right)\right) \\
& \leq \sum_{i=1}^{n} P\left(\left|X_{i}\right|>n^{\alpha}\right) \\
& =n P\left(|X|>n^{\alpha}\right) \longrightarrow 0, \quad n \rightarrow \infty,
\end{aligned}
$$

which implies that

$$
\frac{S_{n}}{n^{\alpha}}-\frac{T_{n}}{n^{\alpha}} \stackrel{P}{\rightarrow} 0 .
$$

Hence, in order to prove (8), we only need to show that

$$
\frac{T_{n}}{n^{\alpha}}-\frac{E T_{n}}{n^{\alpha}} \stackrel{P}{\rightarrow} 0
$$

By (7) again and Toeplitz's lemma, we can get that

$$
\frac{\sum_{k=1}^{n} k^{2 \alpha-2} \cdot k P\left(|X|>k^{\alpha}\right)}{\sum_{k=1}^{n} k^{2 \alpha-2}} \longrightarrow 0, \quad n \longrightarrow \infty .
$$

Note that

$$
\sum_{k=1}^{n} k^{2 \alpha-2} \ll n^{2 \alpha-1}, \quad \text { for } \alpha>\frac{1}{2} .
$$

Combing (35) and (36), we have

$$
n^{-2 \alpha+1} \sum_{k=1}^{n} k^{2 \alpha-1} P\left(|X|>k^{\alpha}\right) \longrightarrow 0, \quad n \longrightarrow \infty .
$$


By Lemma 7 (taking $p=2$ ), (7), and (37), we can get that

$$
\begin{aligned}
& P\left(\left|T_{n}-E T_{n}\right|>\varepsilon n^{\alpha}\right) \\
& \leq C n^{-2 \alpha} E\left|T_{n}-E T_{n}\right|^{2} \\
& \leq C n^{-2 \alpha} \sum_{i=1}^{n} E Y_{n i}^{2} \\
& \leq C n^{-2 \alpha+1}\left[E X^{2} I\left(|X| \leq n^{\alpha}\right)+n^{2 \alpha} P\left(|X|>n^{\alpha}\right)\right] \\
& =C n^{-2 \alpha+1} E X^{2} I \\
& \times\left(|X| \leq n^{\alpha}\right)+C n P\left(|X|>n^{\alpha}\right) \\
& =C n^{-2 \alpha+1} \sum_{k=1}^{n} E X^{2} I \\
& \times\left((k-1)^{\alpha}|X| \leq k^{\alpha}\right)+C n P\left(|X|>n^{\alpha}\right) \\
& \leq C n^{-2 \alpha+1} \sum_{k=1}^{n} k^{2 \alpha} \\
& \times\left[P\left(|X|>(k-1)^{\alpha}\right)-P\left(|X|>k^{\alpha}\right)\right] \\
& +C n P\left(|X|>n^{\alpha}\right) \\
& =C n^{-2 \alpha+1}\left[\sum_{k=1}^{n-1}\left((k+1)^{2 \alpha}-k^{2 \alpha}\right) P\left(|X|>k^{\alpha}\right)\right. \\
& \left.+P(|X|>0)-n^{2 \alpha} P\left(|X|>n^{\alpha}\right)\right] \\
& +C n P\left(|X|>n^{\alpha}\right) \\
& \leq C n^{-2 \alpha+1}\left[\sum_{k=1}^{n} k^{2 \alpha-1} P\left(|X|>k^{\alpha}\right)+1\right] \\
& +C n P\left(|X|>n^{\alpha}\right) \longrightarrow 0, \quad n \longrightarrow \infty .
\end{aligned}
$$

This completes the proof of the theorem.

\section{Acknowledgments}

The authors are most grateful to the Editor Binggen Zhang and anonymous referee for the careful reading of the paper and valuable suggestions which helped in improving an earlier version of this paper. This work was supported by the National Natural Science Foundation of China (11201001, 11171001, and 11126176), the Specialized Research Fund for the Doctoral Program of Higher Education of China (20093401120001), the Natural Science Foundation of Anhui Province (11040606M12, 1208085QA03), the Natural Science Foundation of Anhui Education Bureau (KJ2010A035), the 211 project of Anhui University, the Academic Innovation Team of Anhui University (KJTD001B), and the Students Science Research Training Program of Anhui University (KYXL2012007).

\section{References}

[1] J. Cuzick, "A strong law for weighted sums of i.i.d. random variables," Journal of Theoretical Probability, vol. 8, no. 3, pp. 625-641, 1995.

[2] Z. Bai, P. E. Cheng, and C.-H. Zhang, "An extension of the Hardy-Littlewood strong law," Statistica Sinica, vol. 7, no. 4, pp. 923-928, 1997.

[3] Z. Bai and P. Cheng, "Marcinkiewicz strong laws for linear statistics," Statistics \& Probability Letters, vol. 46, no. 2, pp. 105$112,2000$.

[4] G.-H. Cai, "Marcinkiewicz strong laws for linear statistics of $\rho^{*}$-mixing sequences of random variables," Anais da Academia Brasileira de Ciências, vol. 78, no. 4, pp. 615-621, 2006.

[5] Q. Wu, "A strong limit theorem for weighted sums of sequences of negatively dependent random variables," Journal of Inequalities and Applications, vol. 2010, Article ID 383805, 8 pages, 2010.

[6] S. H. Sung, "On the strong convergence for weighted sums of random variables," Statistical Papers, vol. 52, no. 2, pp. 447-454, 2011.

[7] X.-C. Zhou, C.-C. Tan, and J.-G. Lin, "On the strong laws for weighted sums of $\rho^{*}$-mixing random variables," Journal of Inequalities and Applications, vol. 2011, Article ID 157816, 8 pages, 2011.

[8] X. Wang, X. Li, W. Yang, and S. Hu, "On complete convergence for arrays of rowwise weakly dependent random variables," Applied Mathematics Letters, vol. 25, no. 11, pp. 1916-1920, 2012.

[9] T. K. Chandra and S. Ghosal, "Extensions of the strong law of large numbers of Marcinkiewicz and Zygmund for dependent variables," Acta Mathematica Hungarica, vol. 71, no. 4, pp. 327336, 1996.

[10] T. K. Chandra and S. Ghosal, "The strong law of large numbers for weighted averages under dependence assumptions," Journal of Theoretical Probability, vol. 9, no. 3, pp. 797-809, 1996.

[11] Y. Wang, J. Yan, F. Cheng, and C. Su, "The strong law of large numbers and the law of the iterated logarithm for product sums of NA and AANA random variables," Southeast Asian Bulletin of Mathematics, vol. 27, no. 2, pp. 369-384, 2003.

[12] M.-H. Ko, T.-S. Kim, and Z. Lin, “The Hájeck-Rènyi inequality for the AANA random variables and its applications," Taiwanese Journal of Mathematics, vol. 9, no. 1, pp. 111-122, 2005.

[13] D. Yuan and J. An, "Rosenthal type inequalities for asymptotically almost negatively associated random variables and applications," Science in China. Series A, vol. 52, no. 9, pp. 18871904, 2009.

[14] X. Wang, S. Hu, and W. Yang, "Convergence properties for asymptotically almost negatively associated sequence," Discrete Dynamics in Nature and Society, vol. 2010, Article ID 218380, 15 pages, 2010.

[15] X. Wang, S. Hu, and W. Yang, "Complete convergence for arrays of rowwise asymptotically almost negatively associated random variables," Discrete Dynamics in Nature and Society, vol. 2011, Article ID 717126, 11 pages, 2011.

[16] X. Wang, S. Hu, W. Yang, and X. Wang, "On complete convergence of weighted sums for arrays of rowwise asymptotically almost negatively associated random variables," Abstract and Applied Analysis, Article ID 315138, 15 pages, 2012.

[17] X. P. Hu, G. H. Fang, and D. J. Zhu, "Strong convergence properties for asymptotically almost negatively associated sequence," Discrete Dynamics in Nature and Society, vol. 2012, Article ID 562838, 8 pages, 2012. 
[18] W. Yang, X. Wang, N. Ling, and S. Hu, "On complete convergence of moving average process for AANA sequence," Discrete Dynamics in Nature and Society, vol. 2012, Article ID 863931, 24 pages, 2012.

[19] W. Feller, "A limit theorem for random variables with infinite moments," American Journal of Mathematics, vol. 68, no. 2, pp. 257-262, 1946. 


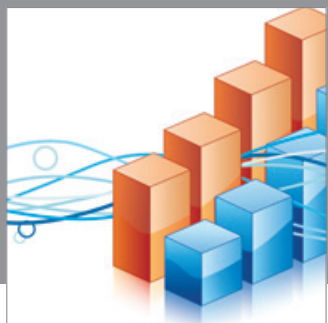

Advances in

Operations Research

mansans

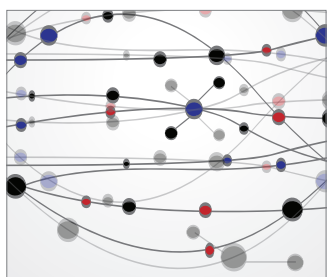

The Scientific World Journal
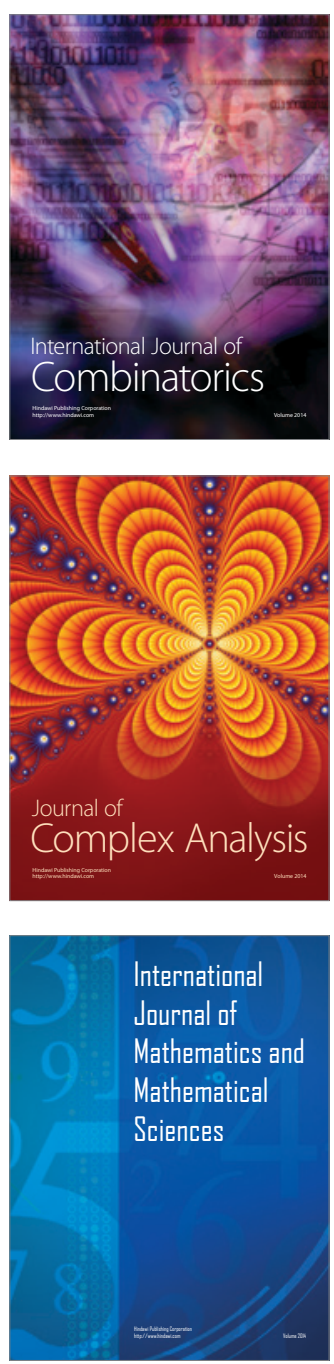
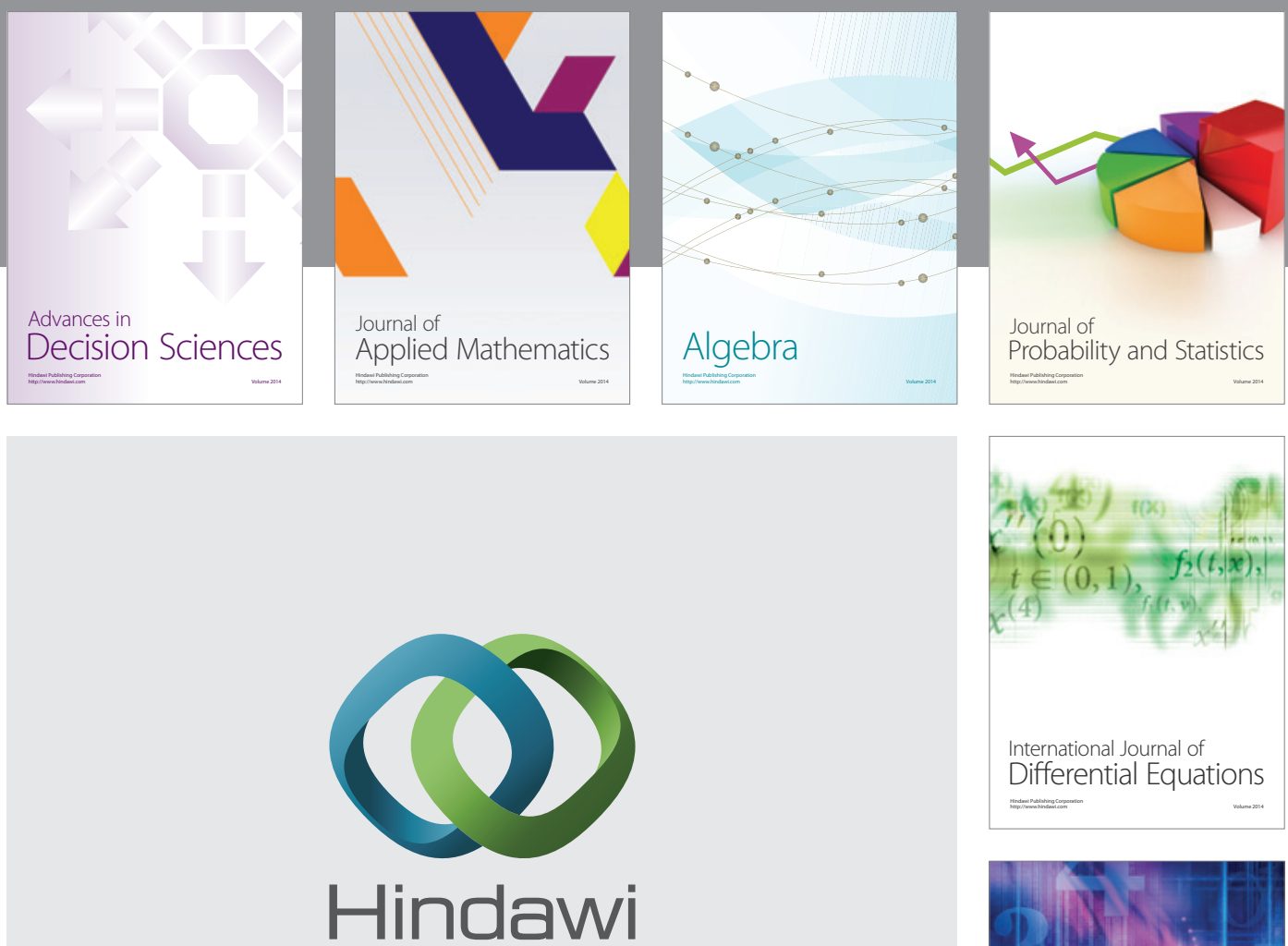

Submit your manuscripts at http://www.hindawi.com
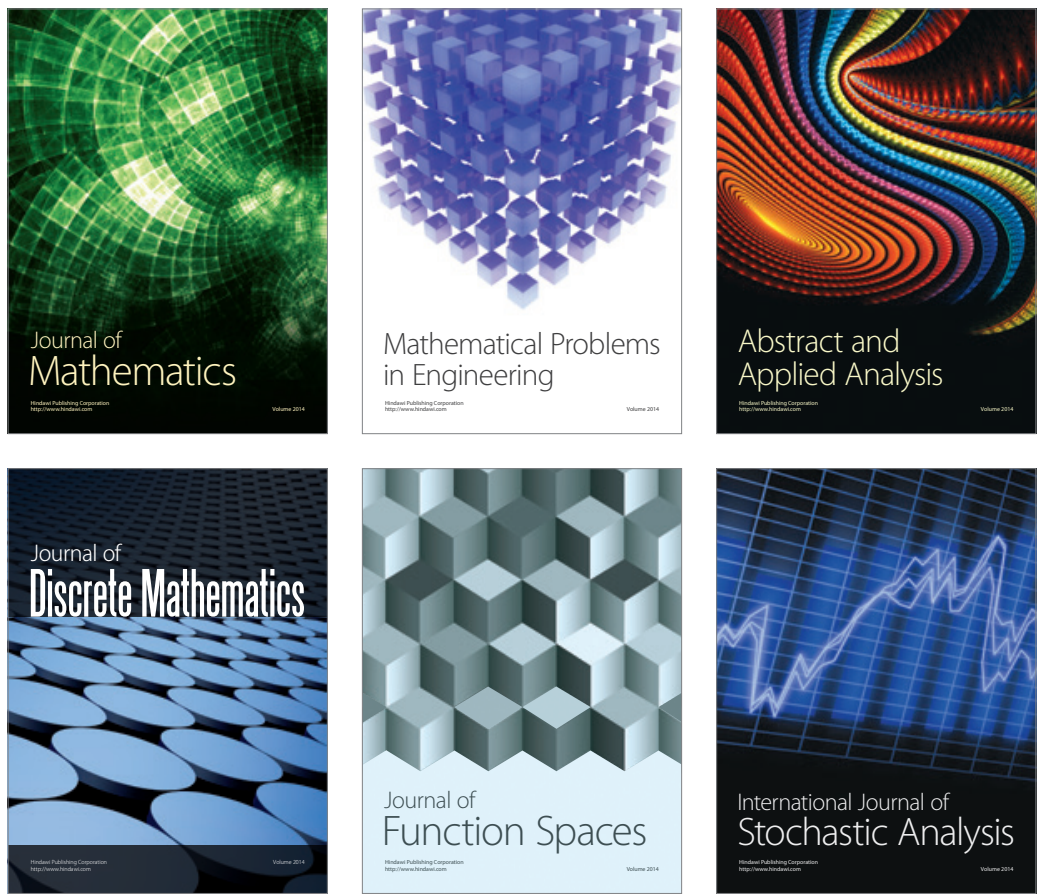

Journal of

Function Spaces

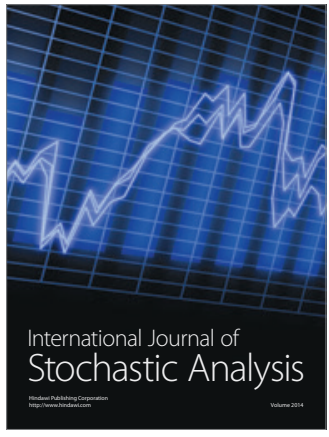

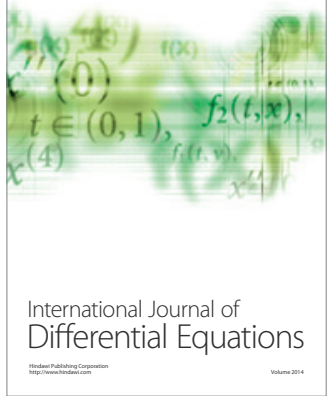
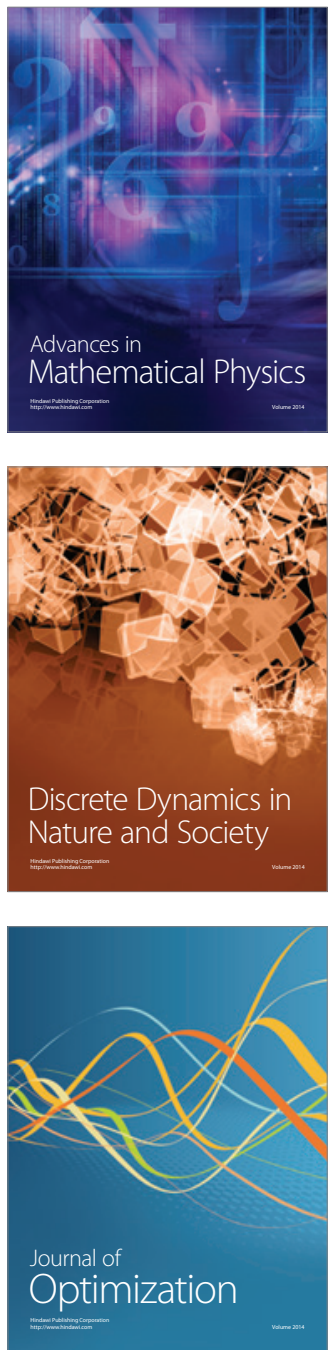